\title{
Evolución e Incidencia de la Enseñanza de Lenguas en la Comunidad Científica
}

\section{Evolution and Incidence of Language Teaching in the Scientific Community}

\author{
Antonio José Moreno-Guerrero ${ }^{1}$ * \\ Ma Aránzazu Fernández-Mora ${ }^{2}$ \\ Magdalena Ramos Navas-Parejo ${ }^{1}$ \\ Carmen Rodríguez-Jiménez ${ }^{1}$ \\ ${ }^{1}$ Universidad de Granada, España \\ ${ }^{2}$ UNED, España
}

\begin{abstract}
El objetivo que se persigue a través de este artículo es determinar el estado actual de la enseñanza de lenguas en el ámbito científico, estableciendo las tendencias en la actualidad atendiendo a diferentes variables como los años de producción, el tipo de producción científica, países y autores más prolíficos, además de instituciones, entre otras cosas. El propósito también es aportar un perfil definido de todo lo relativo a la temática para que suponga una referencia. El método aplicado es un estudio de tipo bibliométrico, realizado mediante la evaluación de rendimiento y la creación de mapas de ciencia, valorando así la evolución conceptual del objeto de estudio. Los resultados de este trabajo expresan el rendimiento de la producción científica y el desarrollo estructural y temático, mostrándose de este modo que la enseñanza de la lengua es una temática que posee un gran impacto e incidencia en la comunidad científica, además de observarse tres temáticas de investigación asentadas en el estudio de la enseñanza de las lenguas, que son el aprendizaje y enseñanza de idiomas basados en tareas, la adquisición de idiomas y la enseñanza-aprendizaje de los mismos.
\end{abstract}

Descriptores: Enseñanza de idiomas; Lengua extranjera; Investigación lingüística; Bibliometría; Base de datos.

The objective of this article is to determine the current state of language teaching in the scientific field, establishing the current trends taking into account different variables such as the years of production, the type of scientific production, more prolific countries and authors, institutions, among others. The purpose is also to provide a defined profile of everything related to the subject so that it is a reference. The applied method is a bibliometric type study, carried out through the evaluation of performance and the creation of science maps, thus assessing the conceptual evolution of the object of study. The results of this work express the performance of the scientific production and the structural and thematic development, showing in this way that language teaching is a subject that has a great impact and incidence in the scientific community, in addition to observing three research topics based on the study of language teaching, which are the learning and teaching of languages based on tasks, the acquisition of languages and the teaching-learning of the same.

Keywords: Language teaching; Foreign language; Language research; Bibliometrics; Database.

*Contacto: ajmoreno@ugr.es

ISSN: 2254-3139

www.rinace.net/riejs/

revistas.uam.es/riejs
Recibido: $\quad 29$ de diciembre 2020

$1^{\text {a }}$ Evaluación: 12 de marzo 2020

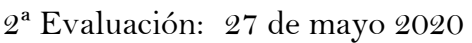

Aceptado: 15 de junio 2020 


\section{Revisión de la literatura}

A continuación se presenta un estudio basado en un análisis pormenorizado de la producción científica sobre la enseñanza de las lenguas en el ámbito científico, en los últimos 10 años, entre 2009 y 2019, de la producción recogida en Web of Science (WoS), aplicando para ello diversas técnicas bibliométricas.

La finalidad de la investigación bibliométrica es determinar el estado actual de la situación de diversas áreas de conocimiento (Quevedo y López, 2010) en nuestro caso, la producción científica sobre la enseñanza de las lenguas. El peso de estos estudios es relevante tanto para las revistas como para los investigadores, ya que buena parte de la producción científica se fundamenta en la difusión y en el impacto de las publicaciones y de los investigadores que en ellas publican (Elkins, Maher, Herbert, Moseley y Sherrington, 2010; Franceschet, 2010), presentando tendencias actuales y orientando futuros estudios en las áreas de investigación (López, Vázquez y Sarasola, 2015; Velasco, Eiros, Pinilla y San Román, 2012;).

Los trabajos sobre la enseñanza de lenguas se centran en unas vertientes tan amplias y diversas, que van desde las ventajas de la utilización de las nuevas tecnologías en la enseñanza-aprendizaje de lenguas extranjeras (Arrosagary, González, Pino y Rodríguez, 2019), ya sean centrándose en los dispositivos móviles (Chang, Sung y Yang, 2015) con una mejora significativa en diversas etapas del aprendizaje o en la enseñanza superior (Crompton y Burke, 2018), en la utilización de la telecolaboración en universidades europeas (Helm, 2015), en la utilización de software determinados como Screencasting (Harper, Green y Fernández, 2018), en las ilimitadas posibilidades del uso de Internet y los medios de comunicación en el uso del lenguaje significativo y auténtico (Richards, 2015), en un enfoque basado en la utilización de podcast para mejorar la pronunciación (Fouz, 2019), en el uso de la tecnología en la enseñanza de una segunda lengua (Tecedor y Campos, 2019), hasta los estudios de la motivación (Lamb, 2017), del metadiscurso (Hyland, 2017), de perfiles de vocabulario de estudiantes de secundaria (Castro, 2017), de uso de ejercicios tradicionales de comprensión auditiva (Cerezo, 2017), hasta un largo etcétera de teorías y métodos de enseñanza y aprendizaje de las lenguas (Asgarin, Kebati y Amirian, 2019; Novakovic y Stojicic, 2019; Rashidi y Hosseini, 2019), que justifican la necesidad de un estudio como el que se presenta.

La evaluación bibliométrica de los contenidos que las revistas presentan, dada la importancia de la difusión del conocimiento para el avance de la ciencia, es un método objetivo y verificable (Velasco et al., 2012), y un indicio claro de calidad de cara a futuras investigaciones (Ruiz, Delgado y Jiménez, 2010; Zych y Buela, 2010).

Por lo que dada la incidencia que muestra la enseñanza de la lengua en la comunidad científica, y con la intención de ofrecer un perfil claro y definido de aquellos que deseen investigar sobre la temática, se proyectan las siguientes cuestiones de investigación: ¿cuál ha sido el rendimiento de la producción científica en el campo de la enseñanza de la lengua en los últimos 10 años en la base de datos WoS?, ¿cuáles son los principales temas de investigación en cuanto a citación e impacto?, ¿cuál es el desarrollo de esos temas?, ¿cómo ha evolucionado el término desde sus inicios en la comunidad científica? 


\section{Método}

La metodología de estudio aplicada es de tipo bibliométrico (Moreno, 2019; Rodríguez, Raso y Ruiz, 2019), mediante el uso de técnicas de rastreo analítico (Álvarez, 2015) y de cuantificación documental (Rodríguez, Trujillo y Sánchez, 2019) mediante una evaluación de rendimiento, en la que se analiza la producción científica de los actores científicos; y la creación de mapas de ciencia, valorando la evolución conceptual del objeto de estudio en un marco longitudinal (Cobo et al., 2011).

Los datos de análisis se han obtenido de la base de datos Web of Science, entre los meses de enero y marzo de 2019. Para la búsqueda se establecieron las palabras clave obtenidas de los tesauros de la UNESCO y ERIC seleccionando: "language education" (LAED), "language instruction" (LAIN) y "language teaching" (LAT), siendo todos ellos elementos clave para el estudio de la enseñanza de idiomas. Seguidamente, se procedió a la descarga de los datos aplicando los siguientes marcadores y operadores booleanos: "language education" OR "language instruction" OR "language teaching". La búsqueda se realiza en el tema, en todas las palabras marcadas en el título, en el resumen y en las palabras claves, comprendiendo el periodo entre 2009 y 2019, es decir, teniendo de referencia los últimos 10 años. En dicha exploración se ha obtenido un total de 10636 referencias.

Para la evaluación de rendimiento y de producción científica se desarrolló un estudio descriptivo, mediante el establecimiento de 9 indicadores, al que se le aplicó una matriz de protocolo PRISMA-P (Hutto, Catalá y Moher, 2016) (cuadro 1) en las siguientes variables: 1.- área de publicación, 2.- año de publicación, 3.- tipo de documentos, 4.organizaciones, 5.- autores, 6.- fuentes de procedencia, 7.- países, 8.- idioma y 9.referencias más citadas, contemplándose en todos los casos todos los documentos posibles.

Cuadro 1. Protocolo PRISMA

\begin{tabular}{ll}
\hline \multicolumn{1}{c}{ INDICADORES } & \multicolumn{1}{c}{ CRITERIO } \\
\hline Área de publicación & Más de 340 en el total \\
\hline Año de publicación & Los 10 últimos años \\
\hline Tipo de documentos & Más de 60 en el total \\
\hline Organizaciones & Más de 40 en el total \\
\hline Fuentes de procedencia & Más de 8 en el total \\
\hline Autores & Más de 330 en el total \\
\hline Países & Más de 85 documentos \\
\hline Idioma & Más de 150 citas \\
\hline Referencias más citadas &
\end{tabular}

Fuente: Elaboración propia.

Para el desarrollo estructural y dinámico del concepto de la enseñanza de las lenguas se ha desarrollado un análisis de co-palabras (Hirsch, 2005), fundamentado en el índice-h y en el número de citas (Cobo, López, Herrera y Herrera, 2011), desarrollando un mapa de ciencia y un análisis de rendimiento para identificar y representar los subdominios conceptuales del campo de investigación y su progreso en la temática de estudio (cuadro 2). El análisis de co-palabras longitudinal, establecido con el programa SciMAT (Cobo, López, Herrera y Herrera, 2012) se estructuró en cuatro fases (Montero et al., 2018):

1. Detección de temas de investigación. De las 10.624 referencias usadas anteriormente, se ha realizado un mapeo para seleccionar aquellas que solamente tienen palabras clave, desechando el resto de los documentos, quedándonos en este caso con un total de 10120 referencias, creando con ello una red de co-ocurrencia mediante 
nodos. La base son las palabras claves localizadas previamente, las cuales se conectan entre sí cuando dos de estas palabras co-aparecen en diversos textos científicos, generando una red normalizada de co-palabras. Mediante el algoritmo de clustering, se han localizado los temas de investigación, mostrándose así las palabras clave fuertemente relacionadas. Se usaron las palabras claves dadas por los autores y las creadas por WoS, según el tipo de documento. Además, se analizó toda la producción científica para comprobar que no hubiera documentos repetidos o que no eran propiamente de la temática tratada, haciendo uso del programa IBM SPSS Statistics 20, el cual, mediante las tablas frecuencias, localiza los documentos duplicados.

2. Representación de temas de investigación. Para ello se ha usado un diagrama estratégico y una red temática (Callon, Courtial y Laville, 1991), formada por dos dimensiones (centralidad y densidad), donde las palabras clave se muestran en cuatro sectores: sector superior derecho, donde alberga los temas motores y fundamentales en la temática de investigación; sector superior izquierdo, donde las conexiones son débiles y son nodos con poca relevancia en la temática; sector inferior izquierdo, donde los temas son relevantes pero no tienen un desarrollo acuciado; y sector inferior derecho, donde los nodos carecen de desarrollo o relevancia, aunque los que aparecen en dicha zona pueden ser temas emergentes.

3. Localización de áreas temáticas. Esto se determina por la evolución cronológica mostrada por los nodos de un periodo a otro. La fuerza de relación se fundamenta según el número de palabras clave que tienen en común. Los periodos establecidos han sido 2009-2012, 2013-2016 y 2017-2019.

4. Análisis de rendimiento. Cada una de las palabras clave, tienen a su vez, una cadena de conexiones que marcan la tendencia de dicho nodo, ofreciendo datos sobre el uso que hace la comunidad científica sobre la misma, para ello se estableció unos protocolos de análisis, establecidos en el cuadro 2.

Cuadro 2. Configuración del análisis de rendimiento

\begin{tabular}{ll}
\hline \multicolumn{1}{c}{ CONFIGURACIÓN } & \multicolumn{1}{c}{ VALORES } \\
\hline Unidad de análisis & Palabras claves autores, palabras clave WoS \\
Tipo de red & Co-ocurrencia \\
Medida de normalización & Índice de equivalencia \\
Algoritmo de clustering & Tamaño máximo: 9; Tamaño mínimo: 3 \\
Medida evolutiva & Índice de Jaccard \\
Medida superpuesta & Índice de inclusión \\
\hline
\end{tabular}

Fuente: Elaboración propia.

\section{Resultados}

A continuación, se exponen los resultados obtenidos a través de la aplicación de rastreo y cuantificación. Los resultados se presentan subdivididos en diferentes categorías que analizan aspectos concretos de la literatura científica y que, por tanto, abordan las diferentes características del objeto de estudio, en este caso la producción científica de la enseñanza de lenguas. 


\subsection{Rendimiento y producción científica}

La combinación de descriptores tal y como ya se ha especificado ofrece un conjunto total de referencias, de las cuales han sido analizados aspectos muy específicos, exponiendo las características de estas referencias y, ofreciendo así, no solo una visión general del conjunto si no una visión parcelada en diferentes parámetros.

\subsubsection{Resultados obtenidos mediante las opciones de búsqueda}

El total de referencias encontradas en la combinación de booleanos es de 10636, habiendo una mayor producción en language teaching que es la que recopila la mayor producción de los cuatro conceptos de búsqueda, mientras que language instruction tiene una incidencia muy baja, tal y como se refleja en la figura 1.

■ language education $\square$ language instruction $\quad$ language teaching

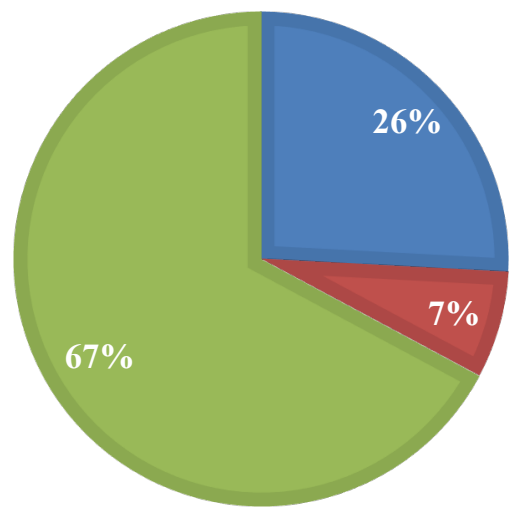

Figura 1. Porcentaje de la producción científica.

Fuente: Elaboración propia.

\subsubsection{Análisis de la producción científica en función al año de publicación}

La producción científica analizada se inicia en el año 2009, teniendo una evolución creciente en los sucesivos años. En los años 2016 y 2017, se observa el mayor incremento de la producción sobre la temática estudiada (cuadro 3).

Cuadro 3. Distribución de la producción científica por año y palabras clave en WoS

\begin{tabular}{cccccc}
\hline \multirow{2}{*}{ AÑO DE PRODUCCIÓN } & \multicolumn{5}{c}{ RESULTADOS } \\
\cline { 2 - 6 } & LAED & LAIN & LATE & Total & $\%$ de 10636 \\
\hline 2019 & 62 & 13 & 110 & 185 & 1,73 \\
2018 & 339 & 85 & 792 & 1.216 & 11,43 \\
2017 & 392 & 91 & 1.028 & 1.511 & 14,20 \\
2016 & 329 & 80 & 1.010 & 1.419 & 13,35 \\
2015 & 337 & 86 & 827 & 1.250 & 11,76 \\
2014 & 327 & 75 & 788 & 1.190 & 11,18 \\
2013 & 232 & 62 & 708 & 1.002 & 9,43 \\
2012 & 204 & 75 & 578 & 857 & 8,06 \\
2011 & 207 & 63 & 573 & 843 & 7,93 \\
2010 & 169 & 65 & 389 & 623 & 5,86 \\
\end{tabular}

Fuente: Elaboración propia. 
Teniendo presente la ley de crecimiento exponencial de la producción científica, tal y como se observa en la figura 2, se muestra una evolución creciente desde el año 2009 hasta el año 2017, en ese preciso instante es donde se produce un ligero descenso en el siguiente año.

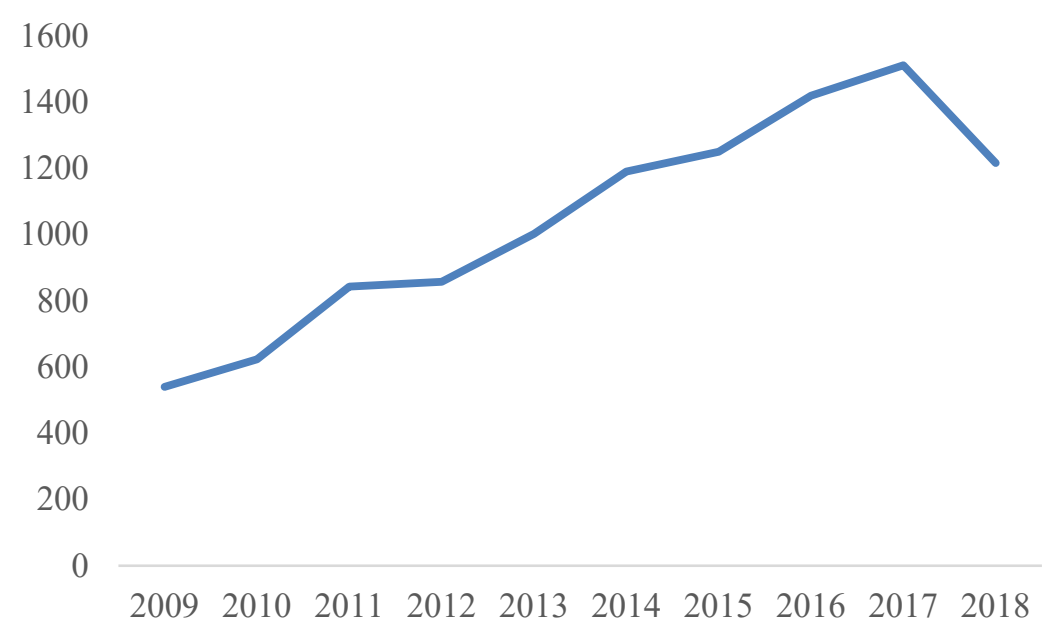

Figura 2. Ley de crecimiento exponencial de la producción científica.

Fuente: Elaboración propia.

\subsubsection{Análisis de la producción científica en función al área de investigación}

El área de investigación en la temática de estudio es Education Educational Research, el cual destaca por encima del resto, seguido de Linguistics. Las demás áreas se sitúan en un nivel parejo muy por debajo de las dos anteriores (cuadro 4).

Cuadro 4. Distribución de la producción científica según el área de investigación

\begin{tabular}{lccccc}
\hline \multirow{2}{*}{ ÁREA DE INVESTIGACIÓN } & \multicolumn{5}{c}{ RESULTADOS } \\
\cline { 2 - 6 } & LAED & LAIN & LATE & Total & \% de 10.636 \\
\hline Education Educational Research & 1.832 & 506 & 4.545 & 6.883 & 64,71 \\
Linguistics & 1.261 & 305 & 2.802 & 4.368 & 41,06 \\
Social Sciences Other Topics & 186 & 31 & 759 & 976 & 9,17 \\
Computer Science & 110 & 49 & 326 & 485 & 4,55 \\
Arts Humanities Other Topics & 61 & 6 & 279 & 346 & 3,25 \\
\hline
\end{tabular}

Fuente: Elaboración propia.

\subsubsection{Análisis de la producción científica en función al tipo de producción}

Los investigadores usan los papers mayoritariamente como tipo de documento para mostrar los resultados de sus estudios de la temática estudiada, seguidos de los proceedings papers. El resto de los documentos apenas tienen relevancia para la propia comunidad científica, según se establece en el cuadro 5 . 
Cuadro 5. Distribución de la producción científica en función al tipo de producción

\begin{tabular}{lccccc}
\hline \multirow{2}{*}{ TiPo PRODUCCIÓN CIENTÍFICA } & \multicolumn{5}{c}{ RESULTADOS } \\
\cline { 2 - 6 } & LAED & LAIN & LATE & Total & \% de 10.636 \\
\hline Papers & 1492 & 672 & 3874 & 6038 & 56,76 \\
Proceedings papers & 519 & 153 & 2348 & 3020 & 28,39 \\
Book chapter & 262 & 44 & 326 & 632 & 5,94 \\
Book review & 200 & 7 & 307 & 514 & 4,83 \\
Editorial Material & 89 & 5 & 123 & 217 & 2,04 \\
Review & 42 & 16 & 64 & 122 & 1,14 \\
Book & 35 & 1 & 32 & 68 & 0,63 \\
\hline$\sum$ & & & & 10611 & 99,73 \\
\hline
\end{tabular}

Fuente: Elaboración propia.

\subsubsection{Análisis de la producción científica en función a las organizaciones de los autores}

La institución que presenta una mayor relevancia, en comparación con el resto de las instituciones, a la hora de generar documentos es Islamic Azad University (cuadro 6).

Cuadro 6. Distribución de la producción científica en función a las organizaciones de los autores

\begin{tabular}{lccccc}
\hline \multirow{1}{*}{\multicolumn{1}{c}{ INSTITUCIONES }} & \multicolumn{5}{c}{ RESULTADOS } \\
\cline { 2 - 6 } & LAED & LAIN & LATE & Total & $\begin{array}{c}\text { \% de } \\
\mathbf{1 0 . 6 3 6}\end{array}$ \\
\hline Islamic Azad University & 23 & 10 & 175 & 208 & 1,95 \\
Kazan Federal University & 50 & 0 & 89 & 139 & 1,30 \\
University of London & 35 & 0 & 60 & 95 & 0,89 \\
Hacettepe University & 22 & 0 & 50 & 72 & 0,67 \\
Education University of Hong Kong Eduhik & 24 & 0 & 49 & 67 & 0,62 \\
University of HradecKralove & 0 & 0 & 60 & 60 & 0,56 \\
University California System & 40 & 16 & 0 & 56 & 0,52 \\
University of Texas system & 32 & 19 & 0 & 51 & 0,47 \\
University of Auckland & 0 & 0 & 44 & 44 & 0,41 \\
Anadolu University & 0 & 0 & 43 & 43 & 0,40 \\
University of Hong Kong & 0 & 0 & 43 & 43 & 0,40 \\
Gazi University & 0 & 0 & 41 & 41 & 0,38 \\
\hline$\sum$ & & & & 919 & 8,57 \\
\hline
\end{tabular}

Fuente: Elaboración propia.

\subsubsection{Autores con mayor producción científica sobre la temática}

$\mathrm{El}$ autor con mayor número de escritos científicos, con un total de 29 es Hubacrova. Le sigue un grupo de tres autores que tienen 15, son Wang Y., Byram y Norton. El resto oscila entre once y nueve textos (cuadro 7 ).

Cuadro 7. Análisis de la producción científica en función de los autores

\begin{tabular}{lccccc}
\hline \multirow{2}{*}{ AUTORES } & \multicolumn{5}{c}{ RESULTADOS } \\
\cline { 2 - 6 } & LAED & LAIN & LATE & Total & \% de 10.636 \\
\hline Hubacrova & 0 & 0 & 29 & 29 & 0,27 \\
Wang Y & 0 & 3 & 12 & 15 & 0,14 \\
Byram & 6 & 0 & 9 & 15 & 0,14 \\
Norton & 6 & 0 & 9 & 15 & 0,14 \\
Mady & 12 & 0 & 0 & 12 & 0,11 \\
Porto & 11 & 0 & 1 & 12 & 0,11 \\
Liu & 0 & 0 & 12 & 12 & 0,11 \\
Borg & 0 & 0 & 11 & 11 & 0,10 \\
Hismanoglu & 0 & 0 & 11 & 11 & 0,10 \\
Laborda & 0 & 0 & 11 & 11 & 0,10 \\
\hline
\end{tabular}




\begin{tabular}{lllccc}
\hline Richards & O & O & 11 & 11 & 0,10 \\
Alemi & 0 & 0 & 9 & 9 & 0,08 \\
Burns & 0 & 0 & 9 & 9 & 0,08 \\
Calik & 0 & 0 & 9 & 9 & 0,08 \\
Wang H & 0 & 0 & 9 & 9 & 0,08 \\
\hline$\sum$ & & & & 190 & 1,74 \\
\hline
\end{tabular}

Fuente: Elaboración propia.

\subsubsection{Análisis de la producción científica en función a la fuente de los textos científicos}

Las revistas que más textos científicos publican sobre la temática de estudio, siguiendo lo marcado en el cuadro 8, destacan por su carácter internacional. Entre todas las revistas sobresale Procedia Social and Behavioral Sciences, seguida de Advances in Social Science Education and Humanities Research.

Cuadro 8. Título de las fuentes que publican sobre la temática de estudio

\begin{tabular}{|c|c|c|c|c|c|}
\hline \multirow[b]{2}{*}{ FUENTE } & \multicolumn{5}{|c|}{ RESULTADOS } \\
\hline & LAED & LAIN & LATE & Total & $\begin{array}{c}\% \text { de } \\
10636\end{array}$ \\
\hline Procedia Social and Behavioral Sciences & 104 & 27 & 502 & 633 & 5,95 \\
\hline $\begin{array}{l}\text { Advances in Social Science Education and } \\
\text { Humanities Research }\end{array}$ & 50 & 0 & 262 & 312 & 2,93 \\
\hline Inted Proceedings & 57 & 8 & 147 & 212 & 1,99 \\
\hline Edulearn proceedings & 31 & 10 & 131 & 172 & 1,61 \\
\hline $\begin{array}{l}\text { Modern Journal of Language Teaching } \\
\text { Methods }\end{array}$ & 19 & $\mathrm{O}$ & 145 & 164 & 1,54 \\
\hline System & 32 & 13 & 108 & 153 & 1,43 \\
\hline Modern Language Journal & 47 & 8 & 73 & 128 & 1,20 \\
\hline ELT Journal & 21 & $\mathrm{O}$ & 81 & 102 & 0,95 \\
\hline Advances in Education Research & 0 & o & 100 & 100 & 0,94 \\
\hline Arab World English Journal & 0 & 6 & 94 & 100 & 0,94 \\
\hline Yazyk I Kultura Language and Culture & 39 & o & 45 & 84 & 0,78 \\
\hline Lecture notes in management Science & 0 & $\mathrm{O}$ & 81 & 81 & 0,76 \\
\hline Foering Language Animals & 38 & 27 & 0 & 65 & 0,61 \\
\hline Porta Linguarum & 48 & 12 & $\mathrm{O}$ & 60 & 0,56 \\
\hline $\begin{array}{l}\text { International Journal of Bilingual Education } \\
\text { and Bilingualism }\end{array}$ & 35 & 7 & $\mathrm{O}$ & 42 & 0,39 \\
\hline$\sum$ & & & & 2408 & 22,58 \\
\hline
\end{tabular}

Fuente: Elaboración propia.

\subsubsection{Análisis de la producción científica en función al país de procedencia de los archivos}

Los países que más textos científicos producen sobre el estudio de la lengua son China y Estados Unidos, considerablemente distanciados del resto de países. El continente que más destaca es América en comparación con el resto (cuadro 9).

Cuadro 9. Distribución de la producción científica en función al país de publicación

\begin{tabular}{lccccc}
\hline \multirow{2}{*}{ PAís } & \multicolumn{5}{c}{ RESULTADOS } \\
\cline { 2 - 6 } & LAED & LAIN & LATE & Total & \% de 10.636 \\
\hline China & 313 & 41 & 13.24 & 1.678 & 15,7 \\
Estados Unidos & 548 & 359 & 691 & 1.598 & 15,02 \\
Inglaterra & 164 & 27 & 406 & 597 & 5,61 \\
España & 84 & 35 & 478 & 597 & 5,61 \\
Rusia & 152 & 10 & 353 & 515 & 4,84 \\
Irán & 59 & 30 & 380 & 469 & 4,4 \\
Brasil & 38 & 7 & 370 & 415 & 3,90 \\
Australia & 152 & 24 & 210 & 386 & 3,62 \\
\hline
\end{tabular}




\begin{tabular}{lccccc}
\hline Canadá & 135 & 43 & 157 & 335 & 3,14 \\
Japón & 158 & 11 & 138 & 307 & 2,88 \\
\hline$\sum$ & & & & 6.897 & 64,72 \\
\hline
\end{tabular}

Fuente: Elaboración propia.

\subsubsection{Análisis de la producción científica en función al idioma de publicación}

El idioma por antonomasia en los documentos generados sobre la temática de investigación es el inglés, destacando por encima del resto, tal y como se muestra en el cuadro 10 .

Cuadro 10. Distribución de la producción científica en función al idioma

\begin{tabular}{lccccc}
\hline \multirow{2}{*}{ IDIOMA } & \multicolumn{5}{c}{ RESULTADOS } \\
\cline { 2 - 6 } & LAED & LAIN & LATE & Total & \% de 10.636 \\
\hline Inglés & 2.410 & 663 & 5.923 & 8.996 & 84,58 \\
Castellano & 43 & 11 & 334 & 388 & 3,64 \\
Portugués & 26 & 5 & 334 & 365 & 3,43 \\
Rusia & 100 & 5 & 188 & 293 & 2,75 \\
Chino & 22 & 10 & 90 & 122 & 1,14 \\
Francés & 15 & 2 & 87 & 104 & 0,97 \\
Alemán & 22 & 12 & 55 & 89 & 0,83 \\
Turquía & 25 & 2 & 50 & 77 & 0,72 \\
\hline$\sum$ & & & & 10.434 & 98,06 \\
\hline
\end{tabular}

Fuente: Elaboración propia.

\subsubsection{Referencias más citadas}

Los documentos publicados en las fechas de 2010 son los más citados de la producción científica sobre la enseñanza de la lengua. El texto de Blackledge y Creese (2010) presenta más del doble de citas que el siguiente autor más citado Heller, que también es del 2010 (cuadro 11).

Cuadro 11. Referencias más citadas en la producción científica

\begin{tabular}{|c|c|c|c|c|}
\hline Autores & AÑo & Título & $\begin{array}{c}\text { FUENTE DE } \\
\text { PROCEDENCIA }\end{array}$ & $\begin{array}{l}\text { NÚMERO } \\
\text { DE CITAS }\end{array}$ \\
\hline $\begin{array}{l}\text { Blackledge y } \\
\text { Creese }\end{array}$ & 2010 & $\begin{array}{l}\text { Translanguaging in the Bilingual } \\
\text { Classroom: A Pedagogy for } \\
\text { Learning and Teaching? }\end{array}$ & $\begin{array}{l}\text { Modern } \\
\text { Language } \\
\text { Journal } \\
\end{array}$ & 481 \\
\hline Heller & 2010 & The Commodification of Language & $\begin{array}{l}\text { Annual Review of } \\
\text { Anthropology }\end{array}$ & 198 \\
\hline $\begin{array}{l}\text { Thorne, } \\
\text { Black y } \\
\text { Sykes }\end{array}$ & 2009 & $\begin{array}{l}\text { Second Language Use, Socialization, } \\
\text { and Learning in Internet Interest } \\
\text { Communities and Online Gaming }\end{array}$ & $\begin{array}{l}\text { Modern } \\
\text { Language } \\
\text { Journal }\end{array}$ & 181 \\
\hline $\begin{array}{l}\text { Norton y } \\
\text { Toohey }\end{array}$ & 2011 & $\begin{array}{l}\text { Identity, language learning, and } \\
\text { social change }\end{array}$ & $\begin{array}{l}\text { Language } \\
\text { Teaching }\end{array}$ & 165 \\
\hline
\end{tabular}

Fuente: Elaboración propia.

\subsection{Desarrollo estructural y temático}

Este epígrafe presenta la evolución de la temática de estudio a través del análisis de las palabras clave y su agrupación en diferentes periodos, así como las obras y citas de cada uno de los periodos y los cambios que han experimentado. 


\subsubsection{Temas de investigación}

Para facilitar la comprensión de la evolución de los estudios en la enseñanza de lenguas se ha organizado la temática en tres periodos: 2009-2012, 2013-2016 y 2017-2019.

La evolución de las palabras clave entre periodos varía, habiendo bastantes salidas y entradas. El paso del primer periodo al segundo supuso la salida de 3.428 palabras clave y la entrada de 7.199, manteniéndose un total de 2.143 palabras clave comunes, habiendo una coincidencia del 38\%. Del segundo al tercer periodo ocurre algo similar, saliendo 6.922 palabras y entrando 4.462, manteniéndose común entre ambos periodos un total de 2.420 , con una coincidencia del $35 \%$, tal y como se muestra en la figura 3.

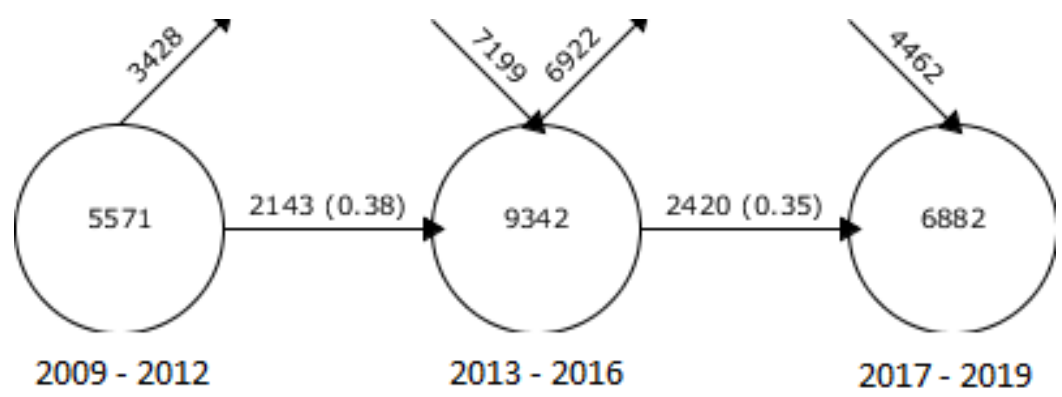

Figura 3. Continuidad de palabras clave entre periodos contiguos Fuente: Elaboración propia.

El primer periodo, comprendido entre las fechas 2009 y 2012, supone la presentación de 7 temáticas principales, siendo classroom y language los que muestran una mayor relevancia, tal y como se observa en los indicadores h, g y q2 en el cuadro 12.

Cuadro 12. Rendimiento temático en el periodo de 2009-2012

\begin{tabular}{lccccc}
\hline NOMBRE & OBRAS & CITAS & ÍNDICE H & ÍNDICE G & ÍNDICE Q2 \\
\hline Classroom & 50 & 896 & 18 & 29 & 23,24 \\
Language & 77 & 1.122 & 18 & 32 & 25,10 \\
Task based language teaching & 15 & 185 & 6 & 12 & 9,49 \\
$\quad$ and learning & 21 & 66 & 5 & 7 & 7,75 \\
Foreing language teaching & 9 & 162 & 5 & 8 & 9,49 \\
Acquisition & 16 & 117 & 7 & 10 & 8,77 \\
Multilingualism & 15 & 142 & 5 & 11 & 11,18 \\
\hline Language teaching and learning & & & & &
\end{tabular}

Fuente: Elaboración propia.

En este periodo, los temas motores son classroom, el cual muestra una mayor centralidad y densidad, y acquisition, con valores menores a la anterior (figura 4). En este caso, ambas son las referencias en la investigación para este periodo. Las investigaciones de classroom, se relacionan con perspective, spanish, 2nd language, comprehension, learner, students, discourse, foreing language. los estudios sobre acquisition se centran en form e instruction.

el tema language teaching and learning puede ser considerado como un tema emergente o que tiende a desaparecer. 


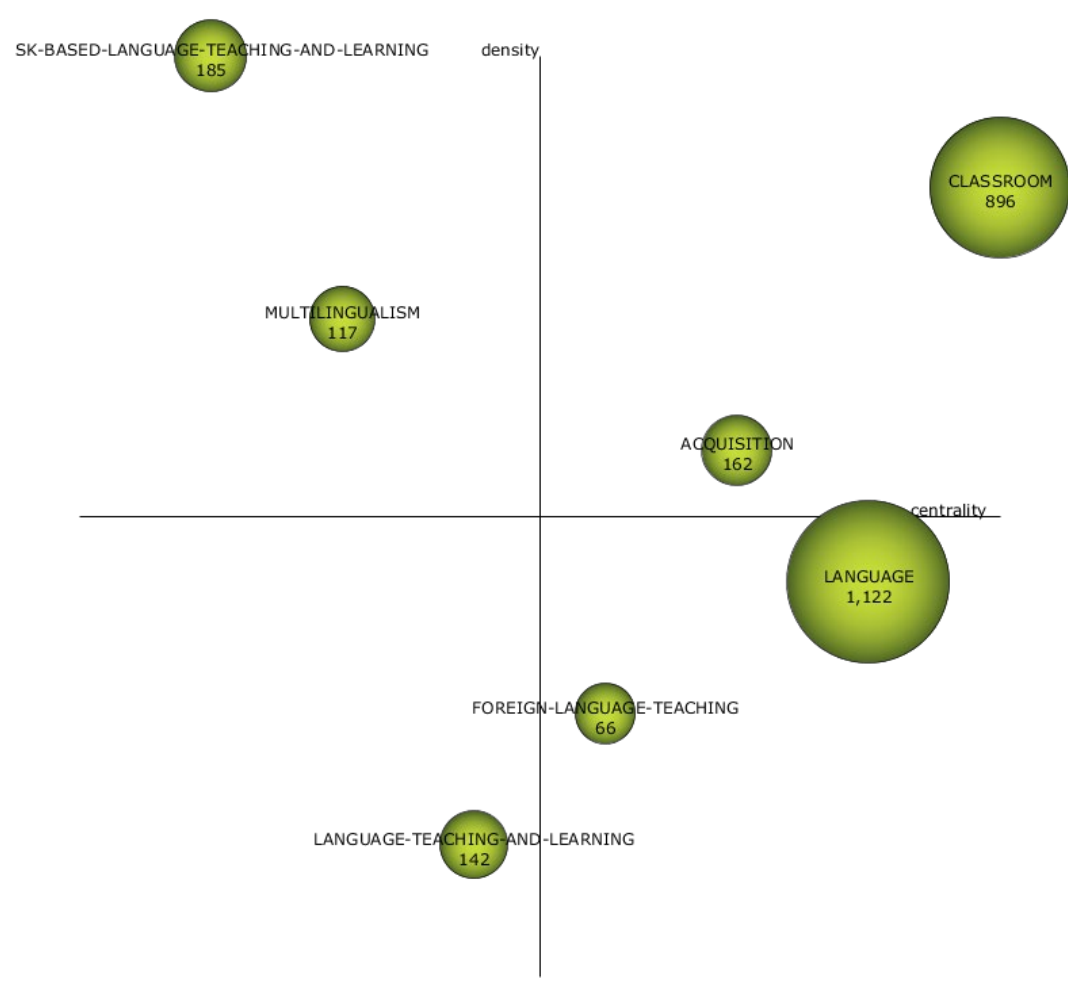

Figura 4. Diagrama estratégico por citas para el periodo $2009-2012$.

Fuente: Elaboración propia.

El segundo periodo, comprendido entre las fechas 2013 y 2016, las temáticas con más relevancia son students y language, siendo la primera la que presenta valores más altos en los indicadores h, g y q2, según se marca en el cuadro 13. En este caso, language coincide con el anterior periodo.

Cuadro 13. Rendimiento temático en el periodo de 2013-2016

\begin{tabular}{lccccc}
\hline \multicolumn{1}{c}{ NoMBRE } & \multirow{2}{*}{ OBRAS } & CITAS & ÍNDICE H & $\begin{array}{c}\text { ÍNDICE } \\
\text { G }\end{array}$ & $\begin{array}{c}\text { ÍNDICE } \\
\text { Q2 }\end{array}$ \\
\hline Acquisition & 47 & 164 & 8 & 9 & 8,94 \\
Students & 165 & 1.046 & 17 & 24 & 21,82 \\
Language & 93 & 333 & 10 & 15 & 13,04 \\
Language teaching and learning & 51 & 104 & 6 & 8 & 8,49 \\
Technology & 34 & 134 & 5 & 11 & 9,75 \\
Motivation & 51 & 228 & 9 & 12 & 10,39 \\
Identity & 36 & 258 & 8 & 15 & 12,00 \\
Foreing language teaching & 31 & 33 & 4 & 4 & 4,47 \\
Multilingualism & 24 & 196 & 6 & 13 & 9,80 \\
Task based language teaching and & 21 & 63 & 4 & 6 & 6,93 \\
$\quad$ learning & & & & & \\
\hline Fuente: Elabor
\end{tabular}

Fuente: Elaboración propia.

En este periodo, los temas motores son students, acquisitions y task based language teaching and learning, tal y como se observa en la figura 5 , de los tres, studens es el que presenta una mayor centralidad, pudiendo ser considerado como el tema con más relevancia, centrando sus estudios en english, education, inmersion, performance, classroom, teacher, children, beliefs. El tema acquisitions desarrolla estudios sobre grammar, corrective feedback, vocabulary, 
perception, errors, recasts y 2nd language. Finalmente, el tema task based language teaching and learning centra sus investigaciones en complexity, fluency, task y needs analysis.

los temas technology y foreign language teaching pueden ser temas emergentes o que tienden a desaparecer.

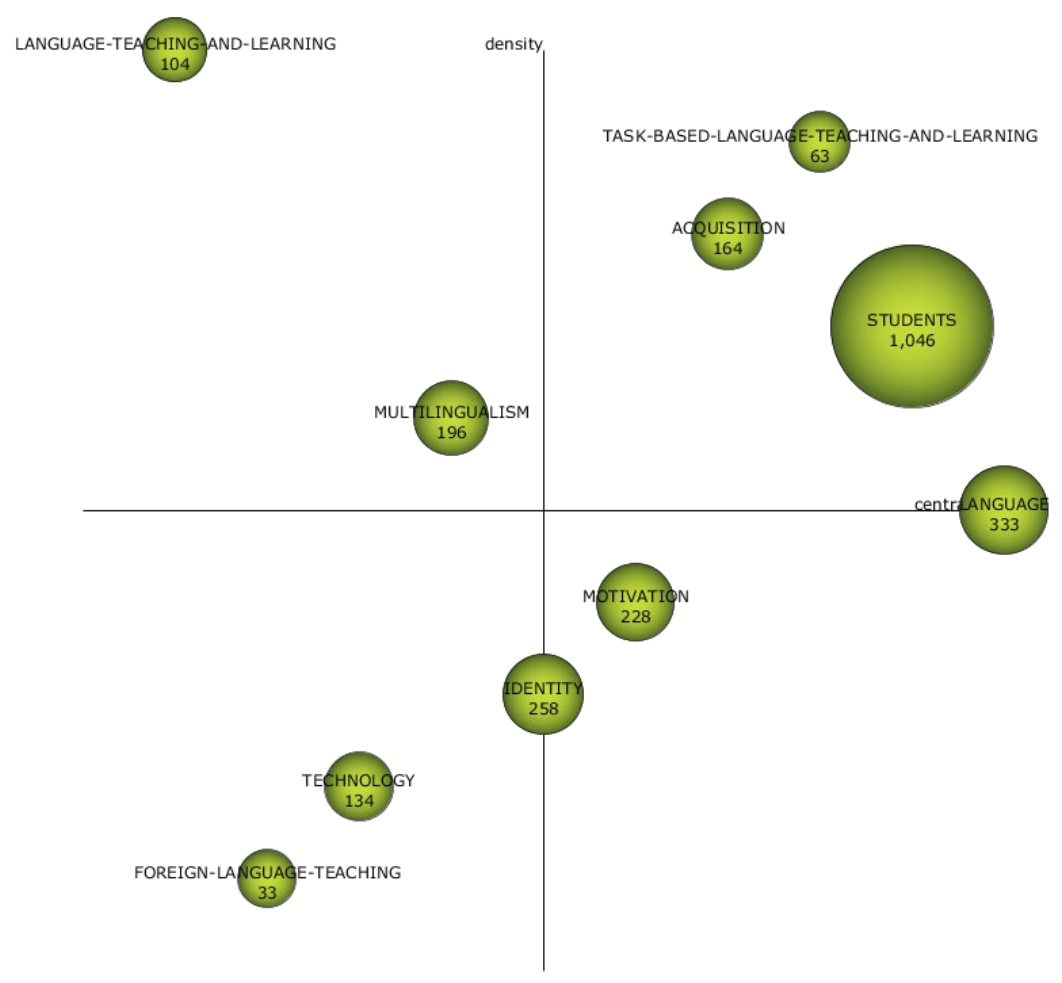

Figura 5. Diagrama estratégico por citas para el periodo $2009-2012$.

Fuente: Elaboración propia.

El último periodo, comprendido entre las fechas 2017 y 2019, muestra que la temática de más relevancia según marcan los indicadores h, g y q2 (cuadro 14), es students (estudiantes), la cual coincide con el anterior periodo.

Cuadro 14. Rendimiento temático en el periodo de 2017-2019

\begin{tabular}{lccccc}
\hline NOMBRE & OBRAS & CITAS & ÍNDICE H & ÍNDICE G & ÍNDICE Q2 \\
\hline Students & 220 & 246 & 7 & 9 & 8,77 \\
Classroom & 34 & 47 & 4 & 5 & 4,00 \\
Language Teaching and learning & 67 & 21 & 2 & 2 & 2,45 \\
Language education & 31 & 19 & 2 & 3 & 3,16 \\
Task based language teaching and & 31 & 8 & 2 & 2 & 2,45 \\
$\quad$ learning & 18 & 11 & 2 & 3 & 3,74 \\
Beliefs & 14 & 15 & 2 & 3 & 2,83 \\
Acquisition & 15 & 7 & 1 & 1 & 1,41 \\
Identity & 13 & 10 & 1 & 2 & 2,45 \\
Bilingual education & 13 & 1 & 1 & 1 & 1,00 \\
Teaching & & & & &
\end{tabular}

Fuente: Elaboración propia.

En este último periodo, el tema motor es students, siendo el único que se encuentra dentro de la sección superior derecha del diagrama, tal y como se muestra en la figura 6. Dicha 
temática centra sus estudios en english, education, comprehension, learner, teacher, attitude, language y learning.

El tema language education (educación lingüística) puede ser considerado como un tema emergente o que tiende a desaparecer.

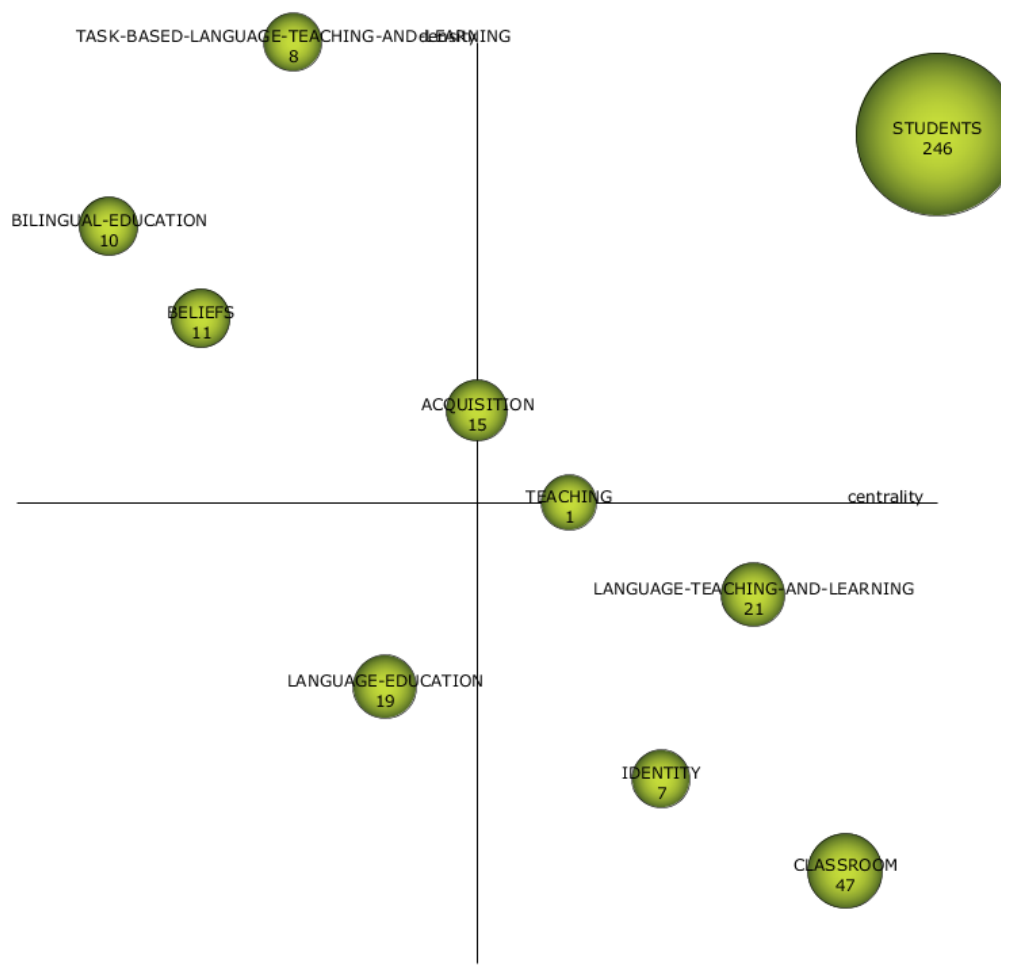

Figura 6. Diagrama estratégico por citas para el periodo 2009-2012 Fuente: Elaboración propia.

\subsubsection{Evolución temática del campo}

Analizando lo mostrado en la figura 7, la evolución de la temática de estudio está definida en toda la producción científica. Las líneas sólidas muestran que los enlaces son propiamente temáticos, mientras que las líneas discontinuas determinan que las relaciones establecidas se deben a que comparten únicamente palabras clave. El tamaño de la temática viene dado por el índice h, por lo que, a mayor tamaño, mayor índice h.

Existen tres temas de investigación que se repiten en los tres periodos con diversa fuerza de relación. Uno de los temas es task based language teaching and learning, cuya conexión temática es alta y al mismo nivel entre los tres periodos. Otro es acquisition, cuya fuerza de conexión temática es adecuada, habiendo más fuerza entre el segundo y tercer periodo que entre el primero y segundo periodo. finalmente, language teaching and learning, cuya conexión temática es media, siendo constante entre periodos.

En el conjunto de los periodos se observan diversas circunstancias. la temática classroom, aparece en el primero y último periodo, pero en el segundo desaparece, generando con ello una brecha conceptual. Hay temáticas que aparecen en el primer y segundo periodo, pero en el tercero desaparecen, tales como foreing language teaching y multilingualism. Otras aparecen en el segundo y tercer periodo, tales como students e identity. Otras en el segundo 
periodo, como es technology. Finalmente, hay temáticas que solamente aparecen en el último periodo, como son language education, beliefs, bilingual education y teaching.

Las conexiones conceptuales establecidas entre las diversas temáticas son mayores que las no conceptuales, lo que muestra líneas de investigación consolidadas entre los diversos periodos.

En el periodo 2009-2012 los temas con mayor índice h son classroom y language. En el periodo 2013-2016, y 2017-2019 el tema con mayor índice h es students.

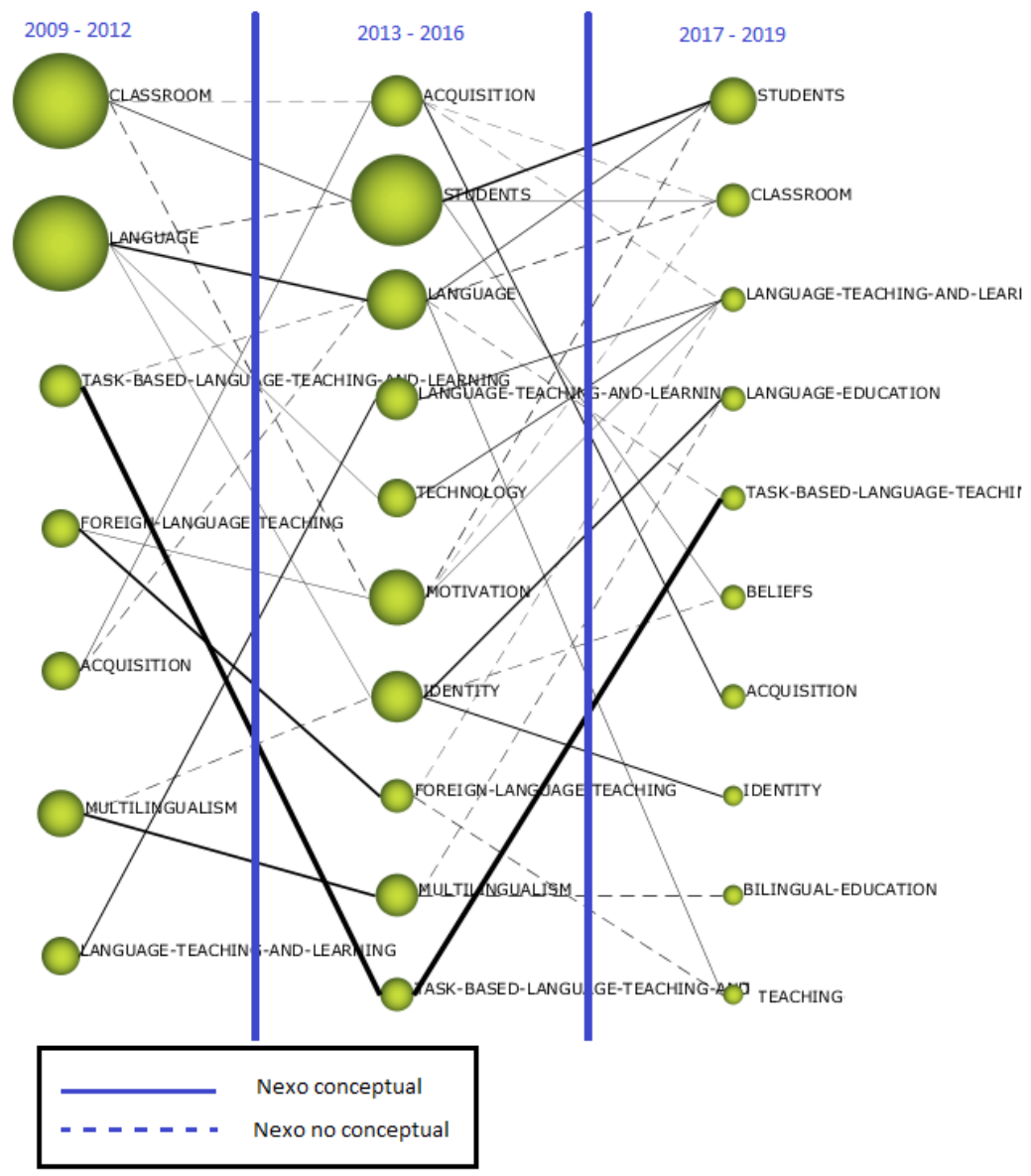

Figura 7. Evolución temática por índice h Fuente: Elaboración propia.

\section{Discusión y conclusión}

La enseñanza de la lengua es una temática de gran impacto y con gran incidencia por parte de la comunidad científica, hecho que queda probado por la gran cantidad de textos científicos recopilados entre los años 2009-2019, con un total de 10.636, siendo la palabra clave "language teaching" la que recopila un total de 7.144 (67\%) producciones. El año con mayor producción es 2017, siendo su tendencia desde los años 2009 hasta dicha fecha alcista, aunque en el año 2018, su incidencia ha descendido a niveles semejantes a los años 2014 y 2015. 
Se comprueba así, cómo la enseñanza de lenguas tiene un lugar preponderante en el ámbito de las publicaciones de carácter científico, independientemente de la vertiente adoptada dentro de este estudio de las diferentes lenguas (Arrosagary et al., 2019; Crompton y Burke, 2018; Rashidi y Hosseini, 2019).

Tal y como se establece tanto en las cuestiones de investigación y como fin último del objetivo general de esta investigación, se aporta una visión general del desarrollo de la temática dentro de la literatura científica. Del mismo modo y siguiendo los aspectos expresados en las citadas cuestiones y en el objetivo de artículos de este corte, son muchos los elementos que se concretan tras la exposición de los resultados, como es el rendimiento de la temática según etapas, la evolución de la misma y estado actual de las temáticas que se desarrollan o surgen a raíz de esta, y componentes inherentes como son autores, tipo de publicaciones, entre otras muchas cosas.

Las áreas de investigación donde más se albergan los estudios sobre esta temática son Education Educational Research y Linguistics, siendo los artículos y las comunicaciones en congresos los medios donde los propios investigadores presentan los resultados de sus estudios, entre los que destacan Procedia Social and Behavioral Sciences y Advances in Social Science Education and Humanities Research. La producción científica es equitativa entre las diversas instituciones que tratan sobre la temática, distinguiéndose Islamic Azad University y Kazan Federal University. Lo mismo ocurre con las instituciones, donde sobresale por encima del resto Hubacrova.

Los países con mayor producción científica son China y Estados Unidos, distanciados del resto, preponderando el inglés de forma muy destacable como idioma utilizado para la presentación de las investigaciones.

Entre los autores más citados se encuentran Creese y Blackledge (2010), Heller (2010), Thorne, Black y Sykes (2009) y Norton y Toohey (2011), cuyas obras son de gran relevancia para la comunidad científica.

Se puede determinar que hay tres temáticas de investigación asentadas en el estudio de la enseñanza de las lenguas, tales como task based language teaching and learning, acquisition, language teaching and learning, teniendo una mayor incidencia el primero.

Después de analizar los datos obtenidos en las diversas temáticas que se recogen en los tres periodos sobre la enseñanza de las lenguas, se puede estipular:

- Classroom: Este tema aparece como tema motor en el primer periodo, pero desaparece en el segundo para volver a incorporarse como tema básico y transversal en el tercer periodo. Durante los años 2009-2012 fue bastante relevante dado que es de los que mayor índice h posee en ese periodo. En el primer periodo presenta un nexo conceptual con students, y no conceptual con motivation y acquisition, del segundo periodo. En cambio, este tema, en el tercer periodo, presenta un nexo conceptual con students, y no conceptual con acquisition y language. Dada la evolución ofrecida durante los tres periodos analizados, se puede determinar que es un tema en decadencia.

- Language: Aparece en el primer y segundo periodo, siendo un tema básico y transversal para la comunidad científica, desapareciendo en el tercero. En el primer periodo es un tema con un alto índice h. Entre el primer y segundo periodo, mantiene una conexión conceptual con language, cuya conexión es 
bastante fuerte, technology e identity, siendo su conexión débil. En cambio, establece una conexión no conceptual con students. Entre el segundo y tercer periodo, establece una conexión con students y teaching, y no conceptual con classroom y task based language teaching and learning. Teniendo presente la evolución en los últimos años, es un tema en decadencia.

- Task based language and teaching: Es uno de los temas que mantiene una continuidad temática en los tres periodos, siendo además su conexión la más alta de todas las establecidas. En el primer periodo, aparece como un tema muy desarrollado y aislado, evolucionando en el segundo periodo a tema motor, para volver a ser un tema muy desarrollado y aislado en el tercer periodo. La conexión que establece con el segundo periodo es conceptual con task based language and teaching y no conceptual con language. en el segundo periodo la relación que establece con el tercero es conceptual con task based language and teaching: Mientras que en el tercer periodo la conexión es conceptual con este mismo tema y no conceptual con language. Analizando la evolución en estos tres periodos, se puede determinar que es un tema en decadencia.

- Foreing language teaching: Es una temática que aparece en el primer periodo como un tema básico y transversal y emergente y aislado en el segundo periodo, no apareciendo en el tercero. La relación entre el primer y segundo periodo es conceptual con foreing language teaching, cuya conexión es media alta, y con motivation. En cambio, la conexión entre el segundo y el tercer periodo es no conceptual con language teaching and learning y teaching. Según se observa en la evolución sufrida en los tres periodos, se puede establecer que es una temática en decadencia.

- Acquisition: Es otro de los temas que mantienen una continuidad temática en el tiempo, con una conexión entre sí baja. En el primer y segundo periodo aparece como un tema motor, pero en el tercero pasa a ser un tema desarrollados y aislado. La conexión establecida entre el primer y segundo periodo es conceptual con acquisition y no conceptual con language. Entre el segundo y tercer periodo mantiene una conexión conceptual con acquisition y no conceptual con language teaching and learning y classroom. Analizando su evolución temporal, se puede determinar que es una temática en decadencia.

- Multilingualism: Es una temática que aparece en el primer y segundo periodo, en ambos casos como tema desarrollado y aislado, pero que desaparece en el tercero. Entre el primer y segundo periodo mantienen una conexión conceptual con multilingualism y no conceptual con identity. entre el segundo y tercer periodo su conexión es no conceptual con language education y bilingual education. Observando la evolución, se puede determinar que es un tema en decadencia.

- Language teaching and learning: Es otro de los temas que aparece en los tres periodos. En el primero es una temática incógnita, dado que puede desarrollarse o puede desaparecer. En el segundo periodo aparece como un tema desarrollado y aislado, mientras que en el tercero emerge como un tema básico y transversal. Entre el primero y segundo periodo mantiene una conexión conceptual consigo mismos, ocurriendo lo mismo entre el segundo y tercer periodo. Y entre el tercer y segundo periodo mantienen una conexión conceptual con language teaching and learning y technology y no conceptual con acquisition y foreign language teaching. 
Dada la evolución temática se puede establecer que es una temática en auge en el ámbito científico.

- Students: Es una temática que aparece en el segundo y en el tercer periodo como tema motor, siendo en ambos periodos el tema con mayor índice h. La conexión entre el segundo y primer periodo es conceptual con classroom y no conceptual con language, entre el segundo y tercer periodo es conceptual con students y biliefs, y entre el tercer y segundo periodo la conexión es conceptual con students y language y no conceptual con motivation. La evolución de la temática es alcista.

- Technology: Es una temática que solamente aparece en el segundo periodo como un tema incógnito, aunque analizando su evolución en los tres periodos se observa que desaparece. Su conexión es conceptual con language en el primer periodo y language teaching and learning en el segundo.

- Motivation: Es una temática que aparece únicamente en el segundo periodo como un tema básico y transversal. Su conexión con el primer periodo es conceptual con foreign language teaching y no conceptual con classroom, mientras que su conexión con el segundo periodo es conceptual con language teaching and learning y no conceptual con classroom y students. Analizando su evolución, se puede determinar que es un tema en decadencia.

- Identity: Es una temática que aparece en el segundo periodo como tema incógnito, y en el tercer periodo se transforma como tema básico y transversal. Su conexión con el primer periodo es conceptual con language y no conceptual con multilingualism, mientras que en el tercer periodo es conceptual con language education e identity y no conceptual con beliefs. Dada su evolución en la temática, se puede determinar que es un tema en auge.

- Language education: Es un tema que aparece en el tercer periodo como tema incógnito, por lo que se no se puede determinar cuál puede ser su evolución en los próximos años. Su conexión con el segundo periodo es conceptual con identity y no conceptual con multilingualism.

- BELIEFS: Es un tema que aparece en el tercer periodo como desarrollado y aislado, no pudiéndose determinar su evolución en los próximos años en el ámbito científico. Su conexión con el segundo periodo es conceptual con students y no conceptual con identity.

- Bilingual education: Es un tema que aparece en el tercer periodo como desarrollad y aislado, no pudiéndose determinar su evolución en los próximos cursos. Su conexión es no conceptual con multilingualism, del segundo periodo.

- Teaching: Es un tema que aparece en el tercer periodo como básico y transversal, no pudiéndose establecer su evolución en los próximos años en el ámbito científico. Su conexión con el segundo periodo es conceptual con language y no conceptual con foerign language teaching.

Finalmente, se puede concluir que la tendencia en los próximos años se centrará en las temáticas languagje teaching and learning, students e identity, siendo una incógnita de cara al futuro las temáticas language education, beliefs, bilingual education y teaching, pudiendo ser los temas de relevancia para la comunidad científica. 
Las limitaciones de la investigación se han centrado principalmente en organizar la red de co-ocurrencia mediante nodos, dado que muchas palabras clave presentaban caracteres incorrectos entre palabras clave que eran en realidad la misma. Como propuesta de mejora, se propone un futuro análisis de aquellas temáticas donde el índice h ha sido menor para ver su incidencia dentro de la temática o su incidencia dentro de los aspectos relacionados de la misma.

Como futuras líneas de investigación, se puede establecer un análisis comparativo con otras bases de datos, tales como Scopus o Google Scholar, para obtener una visión más general de la producción científica de las bases de datos con más relevancia a nivel internacional.

\section{Referencias}

Álvarez, G. (2015). La educación comparada más allá de la REEC: Análisis bibliométrico de la disciplina en las cinco revistas españolas afines de más impacto entre 1995 y 2014. Revista Española de Educación Comparada, 25, 19-45. https://doi.org/10.5944/reec.25.2015.14782

Arrosagary, M., González, M., Pino, M. y Rodríguez, B. (2019). A comparative study of Spanish adult students' attitudes to ICT in classroom, blended and distance language learning modes. Computer E Education, 134, 31-40. https://doi.org/10.1016/j.compedu.2019.01.016

Asgarin, M., Kebati, S. y Amirian, Z. (2019). Interest-based Language Teaching: Enhancing Students' Interest and Achievement in L2 Reading. Iranian Journal of Language Teaching Research, 7(1), 61-75.

Blackledge, A. y Creese, A. (2010). Translanguaging in the bilingual classroom: A pedagogy for learning and teaching? Modern Language Journal, 94(1), 103-115. https://doi.org/10.1111/j.1540-4781.2009.00986.x

Callon, M., Courtial, J. P. y Laville, F. (1991). Co-word analysis as a tool for describing the network of interactions between basic and technological research: The case of polumer chemsitry. Sciencitometric, 22(1), 155-205. https://doi.org/10.1007/BF02019280

Castro, D. (2017). Are we preparing secondary students for a productive use of vocabulary in English as their second language? Porta Linguarum, 28, 141-155.

Cerezo, E. (2017). A critical review of listening comprehension in interpreter training: The case of Spanish translation and interpreting degrees. Porta Linguarum, 28, 7-22.

Chang, K. E., Sung, Y. T. y Yang, J. M. (2015). How effective are mobile devices for language learning? A meta-analysis. Educational Research Review, 16, 68-84. https://doi.org/10.1016/j.edurev.2015.09.001

Cobo, M. J., López, A.G., Herrera, E. y Herrera, F. (2011). Science mapping software tools: Review, analysis, and cooperative study among tools. Journal of the American Society for Information Science and Technology, 62(7), 1382-1402. https://doi.org/10.1002/asi.21525

Cobo, M. J., López, A.G., Herrera, E. y Herrera, F. (2012). SciMAT: A new science mapping analysis software tool. Journal of American Society for Information Science and Technology, 63(8), 1609-1630. https://doi.org/10.1002/asi.22688

Crompton, H. y Burke, D. (2018). The use of mobile learning in higher education: A systematic review. Computers $\Xi^{2}$ Education, 123, 53-64. https://doi.org/10.1016/j.compedu.2018.04.007 
Elkins, M. R., Maher, C. G., Herbert R. D., Moseley, A. M. y Sherrington, C. (2010). Correlation between the journal impact factor and three other journal citation indices. Scientometrics, 85, 81-93. https://doi.org/10.1007/s11192-010-0262-0

Franceschet, M. (2010). Journal influence factors. Journal of Informetrics, 3(4), 239-248. https://doi.org/10.1016/j.joi.2009.12.002

Fouz, J. (2019). Podcast-based pronunciation training: Enhancing FL learners' perception and production of fossilised segmental features. ReCall, 31(2), 150-169. https://doi.org/10.1017/S0958344018000174

Harper, F., Green H. y Fernández, M. (2018). Using screencasts in the teaching of modern languages: investigating the use of jing $(\mathrm{R})$ in feedback on written assignments. Language Learning Journal, 46(3), 277-292. https://doi.org/10.1080/09571736.2015.1061586

Heller, M. (2010). The commodification of language. Annual Review of Anthropology, 39, 101-114. https://doi.org/10.1146/annurev.anthro.012809.104951

Helm, F. (2015). Practices and challenges of telecollaboration in higher education in Europe. Language Learning \& Technology, 19(2), 197-217.

Hirsch, J. E. (2005). An index to quantify an individual's scientific research output. Proceedings of National Academy of Sciences, 102(46), 16569-16572. https://doi.org/10.1073/pnas.0507655102

Hutto, B., Catalá, F. y Moher, D. (2016). La extensión de la declaración PRISMA para revisiones sistemáticas que incorporan metaanálisis en red: PRISMA-NMA. Medicina Clínica, 147(6), 262-266. https://doi.org/10.1016/j.medcli.2016.02.025

Hyland, K. (2017). Metadiscourse: What is it and where is it going? Journal of Pragmatics, 113, 1629. https://doi.org/10.1016/j.pragma.2017.03.007

Lamb, M. (2017). The motivational dimension of language teaching. Language Teaching, 50(3), 301346. https://doi.org/10.1017/So261444817000088

López, E., Vázquez, E. y Sarasola, J. L. (2015). Estudio bibliométrico de Pixel-bit, revista de medios y educación (2000-2013). Píxel-Bit: Revista de Medios y Educación, 46, 65-85. https://doi.org/10.12795/pixelbit.2015.146.05

Moreno, A. J. (2019). Evolución temática de la producción científica de la revista DEDiCA. DEDiCA. Revista de Edcaçao e Humanidades, 16, 189-206.

Montero, J., Cobo, M. J., Gutiérrez, M., Segado, F. y Herrera, E. (2018). Mapeo científico de la Categoría “Comunicación” en WoS (1980-2013). Comunicar, 55(26), 81-91. https://doi.org/10.3916/C55-2018-08

Norton, B. y Toohey, K. (2011). Identity, language learning, and social change. Lenguage Teaching, 44, 412-446. https://doi.org/10.1017/S0261444811000309

Novakovic, A. y Stojicic, V. (2019). Future I tense form in the prizren-timok dialect of Servia. Dialectologia, 22, 101-114.

Quevedo, R. y López, W. (2010). Análisis bibliométrico de las revistas multidisciplinares de psicología incorporadas en la Web of Science (2008-2009). Psicología: Reflexao e Crítica, 23(2), 384-408.

Rashidi, N. y Hosseini, S.A., (2019). The perceptions of iranian policymakers as articulated in iran's foreign language policy document: A systemic functional linguistics approach. Iranian Journal of Language Teaching Research, 7(1), 23-42. 
Rodríguez, A. M., Trujillo, J. M. y Sánchez, J. (2019). Impacto de la productividad científica sobre competencia digital de los futuros docentes: aproximación bibliométrica en Scopus y Web of Science. Revista Complutense de Educación, 30(2), 623-646. https://doi.org/10.5209/RCED.58862

Rodríguez, A. M., Raso F. y Ruiz, J. (2019). Competencia digital, educación superior y formación del profesorado: Un estudio de meta-análisis en la Web of Science. Pixel- Bit. Revista de Medios de Comunicación, 54, 65-81. https://doi.org/10.12795/pixelbit.2019.i54.04

Richards, J. C. (2015). The changing face of language learning: learning beyond the classroom. Relc Journal, 46(1), 5-22. https://doi.org/10.1177/0033688214561621

Ruiz, R., Delgado, E. y Jiménez, E. (2010): Principios y criterios utilizados en España por la Comisión Nacional Evaluadora de la Actividad Investigadora (CNEAI) para la valoración de las publicaciones científicas: 1989-2009. Psicothema, 222, 898-908.

Tecedor, M. y Campos, G. (2019). Developing oral communication in Spanish lower-level courses: The case of voice recording and videoconferencing activities. ReCall, 31(2), 116-134. https://doi.org/10.1017/S0958344018000083

Thorne, S., Black, R. y Sykes, J. (2009). Second language use, socialization, and learning in internet interest communities and online gaming. The Modern Language Journal, 93(1), 802-821. https://doi.org/10.1111/j.1540-4781.2009.00974.x

Velasco, B., Eiros, J. M., Pinilla, J. M. y San Román, J. A. (2012). La utilización de indicadores bibliométricos para evaluar la actividad investigadora. Aula Abierta, 4O(2), 75-84.

Zych, I. y Buela, G. (2010). Internacionalidad de las revistas de psicología multidisciplinar editadas en Iberoamérica e incluidas en la Web of Science. Universitas Psychologica, 9, 27-34. https://doi.org/10.11144/Javeriana.upsy9-1.irpm

\section{Breve CV de los/as autores/as}

\section{Antonio José Moreno Guerrero}

Doctor en el Departamento de Didáctica, Organización Escolar y Didácticas Especiales, dentro del Programa de Modelos Didácticos, Interculturalidad y Aplicación de las Nuevas Tecnologías en las Instituciones Educativas por la Universidad Nacional de Educación a Distancia, Licenciado en psicopedagogía y Diplomado en Educación Especial y en Audición y Lenguaje por la Universidad de Granada. Dispone además de un Máster en informática educativa por la Universidad Nacional de Educación a Distancia y un Experto Universitario en la Dimensión Político-Administrativa de la Inspección Educativa. ORCID ID: https://orcid.orgOOOO-0003-3191-2048. Email: ajmoreno@ugr.es

\section{Ma Aránzazu Fernández Mora}

Profesora tutora del CUID en la especialidad de francés. Licenciada en Derecho por la Universidad de Sevilla y Diplomada en Magisterio en la especialidad de Lenguas Extranjeras por la Universidad de Granada, es además funcionaria de carrera del cuerpo de profesores de Escuelas Oficiales de Idiomas en la especialidad de francés desde 2002. Actualmente se encuentra en el servicio de inspección de educación de la dirección provincial de Ceuta en comisión de servicio. Desde el curso académico 2018/19 ejerce como profesora tutora sustituta del CUID (francés) de la UNED en la ciudad autónoma 
de Ceuta (España). ORCID ID: https://orcid.orgo000-0003-4523-1139. Email: mfernandez@ceuta.uned.es

\section{Magdalena Ramos Navas-Parejo}

Graduada en Óptica y Optometría por la Universidad de Granada y graduada en Magisterio de Educación Primaria por la misma universidad. Máster en Investigación e Innovación en Currículum e Innovación por la Universidad de Granada. Ha recibido una beca de colaboración por el Ministerio de Educación, desarrolladas en el departamento de Didáctica y Organización Escolar de la Universidad de Granada. Actualmente es becaria de investigación con una Ayuda para la Formación del Profesorado Universitario (FPU) por el Ministerio de Ciencia, Innovación y Universidades en el departamento de Didáctica y Organización Escolar en la Universidad de Granada. ORCID ID: https://orcid.org/0000-0001-9477-6325.Email:magdalena@ugr.es

\section{Carmen Rodríguez Jiménez}

Graduada en Educación Primaria por la Universidad de Granada con mención en lengua extranjera (inglés). Máster en Investigación e Innovación en Currículum e Innovación por la Universidad de Granada. Ha recibido becas de iniciación a la investigación por la Universidad de Granada y de colaboración por el Ministerio de Educación de España, desarrolladas en el departamento de Didáctica y Organización Escolar de la Universidad de Granada. Actualmente es becaria de investigación con una Ayuda para la Formación del Profesorado Universitario (FPU) por el Ministerio de Ciencia, Innovación y Universidades en el departamento de Didáctica y Organización Escolar en la Universidad de Granada. ORCID: https://orcid.org/0000-0001-8623-8316. Email: carmenrj@ugr.es 